\title{
Development of a multiplex RT-PCR for simultaneous diagnosis of human metapneumovirus (HMPV) and human respiratory syncytial virus (HRSV) from clinical specimens
}

\author{
Seetha Dayakar ${ }^{1}$ Heera R. Pillai ${ }^{1}$ Vineetha P. Thulasi $^{1} \cdot$ Radhakrishnan R. Nair $^{1}$
}

Received: 29 March 2016/Accepted: 12 September 2016/Published online: 26 September 2016

(C) Indian Virological Society 2016

\begin{abstract}
Human metapneumovirus (HMPV) and human respiratory syncytial virus (HRSV) are ubiquitous respiratory viral pathogens. They belong to the family Paramyxoviridae (subfamily Pneumovirinae) and is responsible for acute respiratory tract infections in children, elderly and immunocompromised patients. We designed and tested a multiplex reverse transcriptase polymerase chain reaction (mRT-PCR) as a cost-effective alternative to real-time PCR and cell culture based detection for HMPV and HRSV. The newly developed PCR was used to screen nasal/throat swab samples from 356 patients with suspected acute respiratory infection attending the Government Medical College, Thiruvananthapuram, Kerala, India. The method was compared with a commercially available kit employing real time PCR, for its sensitivity and specificity. $53(14.9 \%)$ samples were positive for at least one tested pathogen by mRT-PCR. All except one among the positive samples showed similar pathogen profile when tested using real time PCR. $8(15.1 \%)$ among these 53 were positive for HRSVA, $33(62.3 \%)$ positive for HRSVB and 12 (22.6 \%) were positive for HMPV. 17 (32.7\%) samples showed co-infections in them. Sensitivity and specificity of the mRT-PCR was comparable to that of the commercial
\end{abstract}

Electronic supplementary material The online version of this article (doi:10.1007/s13337-016-0348-2) contains supplementary material, which is available to authorized users.

Radhakrishnan R. Nair

radhakrishnan@rgcb.res.in

1 Laboratory Medicine and Molecular Diagnostics, Rajiv Gandhi Centre for Biotechnology, Bio-Innovation Center (BIC), KINFRA Film \& Video Park, Thiruvananthapuram, Kerala 695585, India kit. Our findings indicate that this newly developed mRTPCR can be used as a cost-effective alternative for laboratory diagnosis of HMPV/HRSV infection and will significantly reduce diagnostic costs for these viruses in clinical settings.

Keywords Acute respiratory infection · Human metapneumovirus - Human respiratory syncytial virus . Multiplex reverse transcriptase PCR - Real-time reverse transcriptase PCR

\section{Introduction}

Globally, acute respiratory infections (ARI) are reported to account for approximately $20 \%$ of total deaths in children under 6 years of age [26]. Human respiratory syncytial virus (HRSV) and human metapneumoviruses (HMPV) are negative sense single-stranded RNA viruses and are the main causative agents of ARI in humans worldwide across all age groups $[1,14,23]$. The real challenge in treating these infections is accurate and low cost early diagnosis suitable for use in resource poor settings (http://www.jhsph.edu/ research/centers-and-institutes/ivac/resources/IVAC-2015Pneumonia-Diarrhea-Progress-Report.pdf) [18].

Real time reverse transcriptase polymerase chain reaction (rRT-PCR) has replaced less sensitive and time consuming techniques like viral culture and serology for diagnosis of viral respiratory diseases in adults $[7,15,21]$. However, costs for doing such tests sometimes hinder accurate clinical diagnosis due to the ever increasing number of respiratory viruses with a repertoire of causing clinically indistinguishable disease phenotype. Currently, diagnosis of human respiratory syncytial virus (HRSV) and Human metapneumovirus (HMPV) relies on the molecular 
detection of viral RNA using rRT-PCR and they have overlapping clinical symptoms and are also seen to coinfect [25]. Thus we developed a multiplex reverse transcriptase polymerase chain reaction (mRT-PCR) assay for the detection of HMPV/HRSV from clinical specimens as a cost-effective alternative and compared its efficacy with established rRT-PCR. We found the newly developed mRT-PCR to have great potential for use in epidemiological vigilance and clinical diagnosis with comparable specificity and sensitivity to that of rRT-PCR $[4,8,11,27]$.

The primary objective of the present study was to analyze acute respiratory tract infections from children between 0 and 6 years of age and adults above 50 years of age. Samples were collected from June 2014 to November 2015 from patients in south India.

\section{Materials and methods}

\section{Study design and sample collection}

Nasal/throat swabs were collected from clinically suspected ARI (anorexia, bronchiolitis, cough, diarrhea, diffuse wheezes and rales, dyspnea, fever, hoarseness, myalgia, rhinitis, sore throat, sputum with COPD and vomiting) subjects at Government Medical College, Thiruvananthapuram, Kerala, India. After informed consent prior to enrollment, 356 patients were sampled by gently rubbing the nasal turbinate/throat cavity with a moistened cotton tip swab and placing it in $5 \mathrm{ml}$ of HiViral transport kit (Himedia Laboratories, India). Specimens were transported to the laboratory in cold at $4{ }^{\circ} \mathrm{C}$ and stored at $-70{ }^{\circ} \mathrm{C}$ until processing. All rRT-PCR and mRT-PCR assays for HMPV/ HRSV were performed on same freeze-thawed aliquots.

\section{Total nucleic acid and viral RNA extraction}

Total nucleic acid for rRT-PCR analysis was isolated from nasal/throat swab samples using the recommended SpinStar nucleic acid kit (ADT Biotech, Malaysia) according to the manufacturer's recommendation. Viscous samples were pretreated with kit provided mucolytic agent prior to extraction. QIAamp Viral RNA Mini Kit was used to extract total RNA for the mRT-PCR analysis. All samples were also tested for GAPDH in the same mRT-PCR assay to check for adequate recovery of sample RNA and the absence of RT-PCR inhibitors.

\section{mRT-PCR}

The primers for the mRT-PCR (Supplementary 02) were designed from consensus genome regions obtained from Gen Bank (http://www.ncbi.nlm.nih.gov/) for each virus

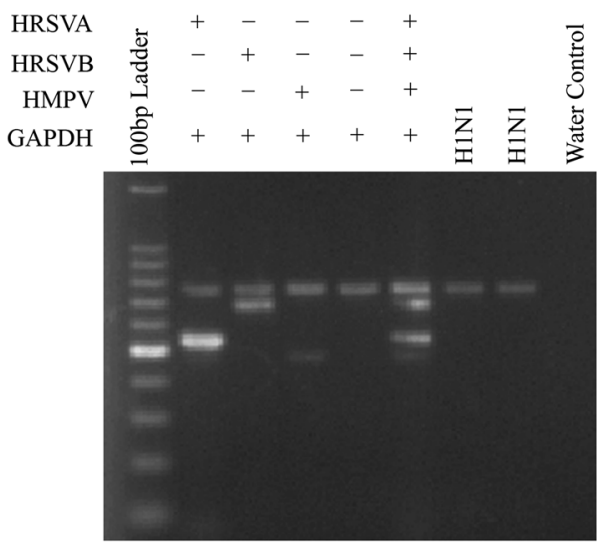

Fig. 1 Agarose gel analysis of PCR products from mRT-PCR of HRSV and HMPV from clinical samples. Two H1N1 positive clinical samples were used as controls for testing primer specificity

and procured from Sigma Aldrich (Bangalore, India). The primers for HRSVA and B were designed on the evolutionarily conserved nucleoprotein gene and for HMPV on the matrix gene. $1 \mu \mathrm{g}$ of RNA template was used for each PCR reaction using the Super Script III One-Step RTPCR System. Each reaction also contained $2 \mu \mathrm{l}$ each of RSVA Forward $(10 \mu \mathrm{M})$, RSVA Reverse $(10 \mu \mathrm{M})$, RSVB Forward $(10 \mu \mathrm{M})$, RSVB Reverse $(10 \mu \mathrm{M})$, HMPV Forward $(10 \mu \mathrm{M})$ and HMPV, Reverse $(10 \mu \mathrm{M})$ primers; $25 \mu \mathrm{l} 2 \times$ reaction mix, $2 \mu \mathrm{l}$ superscript III RT/Platinum Taq mix (Invitrogen, Life technologies) in a final volume of $50 \mu \mathrm{l}$. Negative (water) and positive (rRT-PCR confirmed patient samples) controls were run with each experiment. PCR reactions were performed using the following reaction conditions in Gene Amp PCR system 9700 (Applied Biosystems, USA): First $30 \mathrm{~min}$ at $55^{\circ} \mathrm{C}$ and then $2 \mathrm{~min}$ at $95{ }^{\circ} \mathrm{C}$ to inactivate the reverse transcriptase; this was followed by PCR amplification with initial denaturation step at $95^{\circ} \mathrm{C}$ for $15 \mathrm{~s}$ and then 40 cycles of denaturation at $95{ }^{\circ} \mathrm{C}$ for $15 \mathrm{~s}$, annealing at $55^{\circ} \mathrm{C}$ for $30 \mathrm{~s}$, extension at $68{ }^{\circ} \mathrm{C}$ for $1 \mathrm{~min}$ followed by a final extension step at $68{ }^{\circ} \mathrm{C}$ for $5 \mathrm{~min}$. Amplified products were electrophoresed in $2 \%$ agarose gels and stained with ethidium bromide solution for visualizing using the Syngene Gbox (Syngene, USA) gel documentation system (Fig. 1).

\section{rRT-PCR}

The rRT-PCR assays for single tube detection of HRSVA and HRSVB was done using the RealStar RSV RT-PCR Kit 1.0 (Altona diagnostics GmbH, Germany). RealStar hMPV RT-PCR Kit 1.0 (Altona diagnostics GmbH, Germany) was used to detect HMPV from the clinical samples. Twenty microliters of extracted eluent was used as a template for PCR amplification for both the rRT-PCR 
assays following manufactures instruction using the Rotor gene 5plexHRM real-time platform (QIAGEN, Germany) (Fig. 2a, b).

\section{Nucleotide sequencing and phylogenetic analysis}

Representative PCR products from the mRT-PCR was confirmed using Sanger sequencing using the same forward primers used in the PCR. The reverse primer was used in cases where the forward primer reaction failed for the sequencing protocol. Agarose gel purified fresh PCR products were subjected to cycle sequencing using the Big Dye Terminator v3.1 Cycle Sequencing Kit (Applied Biosystems, USA) according to the manufacturer's instructions. Sequences were read using the ABI PRISM 7900 sequencer (Applied Biosystems, USA) and results were analyzed using sequencing analysis 5.2 software (Applied Biosystems, USA). The obtained sequences were subjected to the NCBI BLAST analysis (http://blast.ncbi. nlm.nih.gov/) for sequence homology analysis. Sequences obtained from the current study subjects were aligned with representative global sequences reported in other studies for construction of phylogenetic tree to check for strain relatedness, using the Molecular Evolutionary Genetic Analysis (MEGA 6.0) software [20].

\section{Statistical analysis}

Statistical analysis was carried out using Epitools (Epitools epidemiological calculators, AusVet Animal Health Services; http://epitoolsausvet.com.au) and agreement between the two tests was determined using concordance and kappa of agreement. Both concordance and kappa coefficients measures the agreement between two variables and provides a more robust measure than simple percent agreement. The sensitivity and specificity of one test with reference to the other and vice versa was also calculated. Analytical sensitivity of the multiplex assay was determined by testing serial dilutions of the quantified RNA for each target. Experiments were repeated five times to check for consistent diagnosis for each virus.

\section{Results}

\section{Characteristics of patients and performance of mRT-PCR, rRT-PCR}

Clinical manifestations recorded at the time of sample collection (Supplementary 01) was indistinctive for HRSV or HMPV associated illness. 53 (14.9\%) samples were
Fig. 2 Representative amplification curves obtained with the HRSV (a) and HMPV (b) rRT-PCR. Each reaction also had an internal control (not shown)
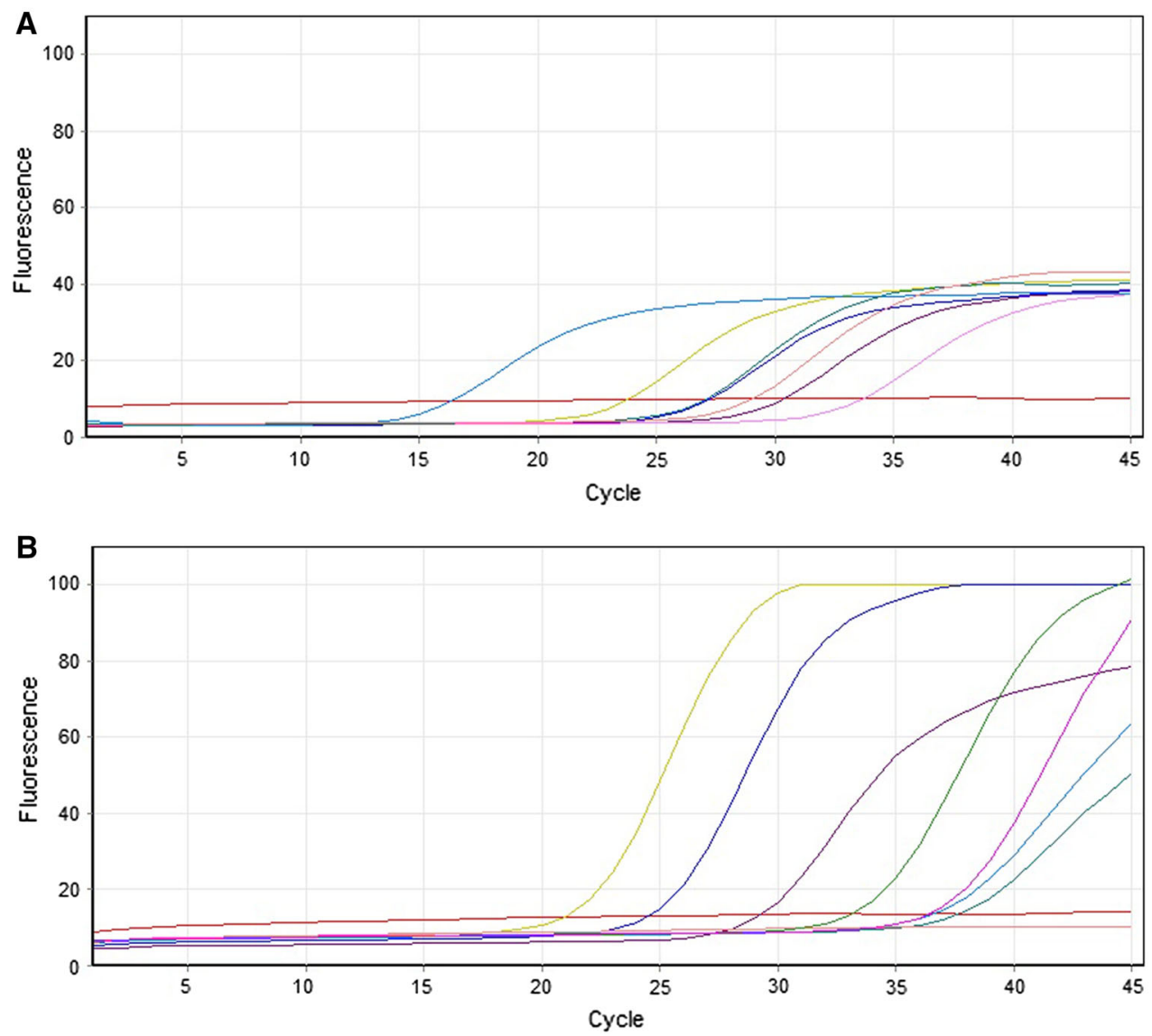
positive for at least one tested pathogen among the 356 subjects tested by mRT-PCR (Table 1). The same samples were also positive for the detected pathogen by mRT-PCR when tested using rRT-PCR, except one which was only detected by mRT-PCR. $8(15.1 \%)$ among these 53 were positive for HRSVA, $33(62.3 \%)$ positive for HRSVB and $12(22.6 \%)$ were positive for HMPV. 17(32.1\%) samples showed co-infections in them. $70 \%$ (29 samples) of all samples positive for HRSV $(\mathrm{n}=41)$ and $67 \%$ (8 samples) of all samples positive for HMPV $(n=12)$ belonged to the $<6$ years age group when tested by both methods (Table 1).

\section{Sensitivity and specificity of mRT-PCR/rRT-PCR}

The overall concordance between both the methods was $99.72 \%$ and kappa correlation was 0.989 . The concordance and kappa coefficients for detection of individual viral pathogens compared to rRT-PCR were as follows: HRSVA $100.0 \%, 1.000$; HRSV B 99.72 \%, 0.983; HMPV $100.0 \%$, 1.000 respectively (Table 1 ). Sensitivity and specificity of mRT-PCR were overall 100.0 and $99.6 \%$; respectively, and for individual pathogens were HRSVA 100.0, $100.0 \%$; HRSVB 100.0, $99.6 \%$; HMPV 100.0, $100.0 \%$ respectively when rRT-PCR detection was considered as the gold standard (Table 2). On the other hand sensitivity and specificity of rRT-PCR were over all 98.1 and $100.0 \%$ respectively when mRT-PCR was taken as the gold standard. Individual pathogens then showed the following sensitivity and specificity respectively: HRSVA 100.0, $100.0 \%$; HRSVB 96.9, $100.0 \%$; HMPV 100.0, $100.0 \%$ (Table 2).

\section{Sequencing and phylogenetic analysis}

The BLAST data confirmed the amplified products to be that of HRSV and HMPV. The representative HRSVA sequences clustered closely with strains from India (Accession: KC731483), China (Accession: KC978856) and USA (Accession: KJ627738) at minimum $98 \%$ identity or above, whereas HRSVB sequence was clustered with Vietnam isolates (Accession: KJ939933) at $97 \%$ identity. The HMPV sequence was clustered with an isolate from Singapore (Accession: JQ307647) at $99 \%$ identity (Figs. 3, 4).

\section{Discussion}

Major respiratory viral pathogens recorded globally are influenza virus, rhino virus, corona virus, human respiratory virus and metapneumovirus $[2,3,13,17,22]$. There have been very few studies which have attempted for rapid diagnosis of respiratory viruses using multiplex PCRs [5]. Use of molecular tests in diagnosis of important respiratory viral causative agents such as HRSV/HMPV has gained wide acceptance. The diagnosis of HMPV is even tougher, as the virus is difficult to isolate in cell culture. Considering the necessity of rapid diagnosis for the clinician, virus isolation on cell culture has been replaced by molecular diagnostic tests. rRT-PCR method for reliable diagnosis of HMPV from respiratory secretions is currently the test of choice. Few studies have developed and evaluated multiplex PCR assays with the aim of providing a tool capable of detecting complete panel of known respiratory viruses [16].

HMPV infection acts as an enhancer of chronic obstructive pulmonary disease and concurrently patients with COPD are more prone to HMPV infection. Studies to date have also shown a significant correlation between HRSV infection and development of childhood asthma in children experiencing RSV bronchiolitis during the first decade of life as opposed to older children. Clinical data from our study also probably points to the fact that a family history of asthma could be a significant non-modifiable risk factor for HRSV infection (Supplementary 01) [9].

In addition, recent studies report that RSV RNA can be identified in patients with chronic obstructive pulmonary disease during periods of exacerbations and during asymptomatic periods at similar rates [10]. Thus it warrants for development of cost-effective and rapid tests like our mRT-PCR for screening of asymptomatic carriers in vulnerable populations and outbreak scenarios (Supplementary 01$)$.

To the best of our knowledge, this is the first study from southern India employing a single step mRT-PCR for detection of HMPV and HRSV directly from clinical samples. Confirmation of HMPV/HRSV etiology by this method gives prompt response in $<24 \mathrm{~h}$, which is important for therapy and critical for avoiding unwanted use of antibiotics. mRT-PCR of nasopharyngeal aspirates or
Table 1 Comparative analysis of real-time RT-PCR and inhouse multiplex RT-PCR

\begin{tabular}{|c|c|c|c|c|c|c|}
\hline \multicolumn{5}{|c|}{ Sample results in real time RT-PCR versus in house multiplex RT-PCR } & \multirow[t]{2}{*}{ Concordance $(\%)$} & \multirow[t]{2}{*}{ Kappa } \\
\hline Virus & $+/+$ & $+1-$ & $-1+$ & $-1-$ & & \\
\hline Overall & 52 & 0 & 1 & 303 & 99.72 & 0.989 \\
\hline HRSV A & 8 & 0 & 0 & 348 & 100.00 & 1.000 \\
\hline HRSV B & 32 & 0 & 1 & 323 & 99.72 & 0.983 \\
\hline HMPV & 12 & 0 & 0 & 344 & 100.00 & 1.000 \\
\hline
\end{tabular}


Table 2 Evaluation of real time RT-PCR and multiplex RT-PCR

\begin{tabular}{|c|c|c|c|c|}
\hline \multirow[t]{2}{*}{ Virus } & \multicolumn{2}{|c|}{ rRT-PCR versus mRT-PCR as gold standard } & \multicolumn{2}{|c|}{ mRT-PCR versus rRT-PCR as gold standard } \\
\hline & Sensitivity (\%) & Specificity (\%) & Sensitivity (\%) & Specificity (\%) \\
\hline Over all & 98.10 & 100.00 & 100.00 & 99.60 \\
\hline HRSV A & 100.00 & 100.00 & 100.00 & 100.00 \\
\hline HRSV B & 96.90 & 100.00 & 100.00 & 99.60 \\
\hline HMPV & 100.00 & 100.00 & 100.00 & 100.00 \\
\hline
\end{tabular}

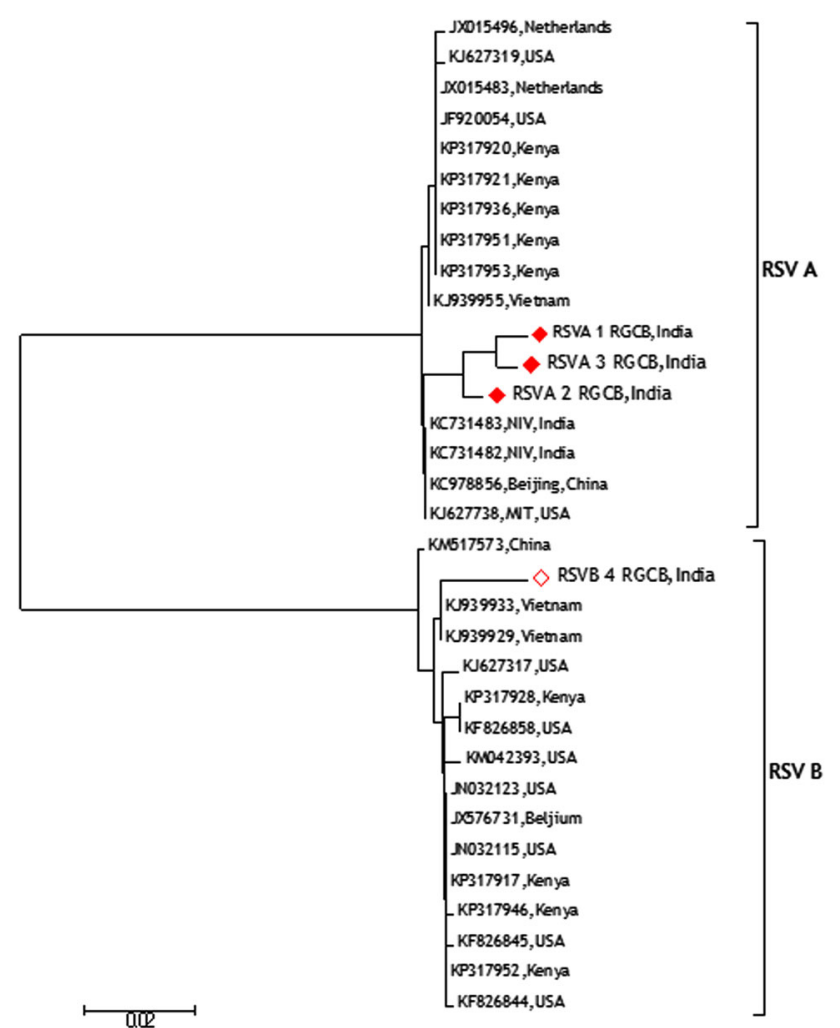

Fig. 3 Neighbor joining tree representing the phylogenetic relationship of the sequences from RGCB RSV, India isolates with partial genome sequences of HRSVA and B isolates in the NCBI database

swabs can be considered a reliable method to establish the diagnosis of HMPV/HRSV infection from clinical samples. Multiplex platforms that allows concurrent detection of several respiratory viruses have the advantage of providing specific results with a rapid turnaround time, and are becoming increasingly viable for commercial use [5].

Viral co-infection has been described previously in $5-20 \%$ of infected patients in different studies [6, 24]. The mRT PCR method also detected a significant proportion of samples to have co-infections from both viruses (17 of the 53 positive samples). Concordance between both the methods was $100 \%$ for HRSV A and HMPV; and $99.7 \%$ for RSV B. There are contradictory reports on the association between HRSV and HMPV co-infection and disease severity. Some studies have found that co-infection leads to an increased rate of intensive care unit admittance, but

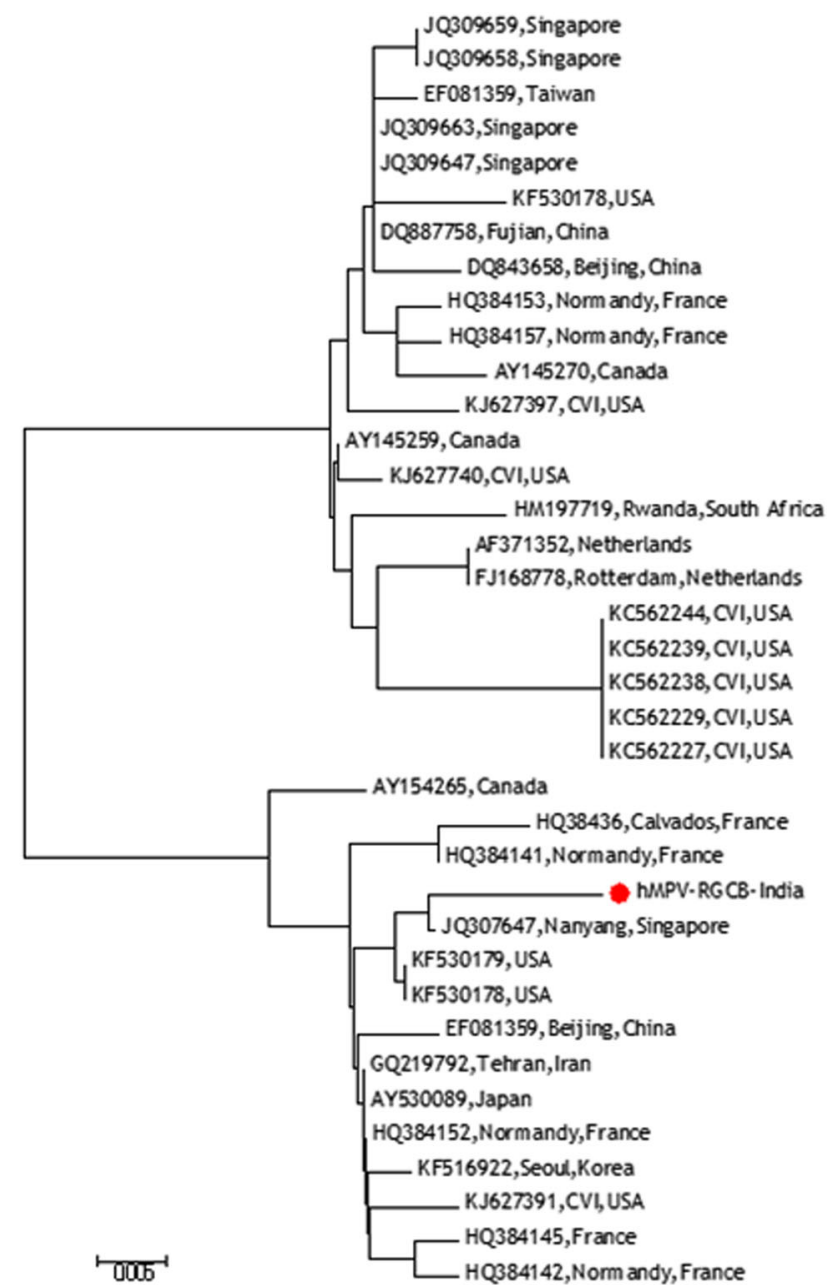

Fig. 4 Neighbor joining tree representing the phylogenetic relationship of the sequences from RGCB HMPV, India isolates with partial genome sequences of HMPV isolates in the NCBI database

others found no association between co-infection and disease severity [19]. However, our patient population were primarily $<6$ years children and $>50$ years adults. Therefore larger studies of chronically ill and elderly patients will be needed before conclusions may be generalized to all patient subgroups.

While mRT-PCR assay may be more economical due to the fact that multiple pathogens can be detected in a single assay without a proportional increase in reagent costs, they have their drawbacks as well. Their detection sensitivities 
are often lower than those of equivalent mono-plex PCR assays. Thus we carefully designed our mRT-PCR to have detection sensitivities comparable to such mono-plex assays and produce reproducible results. Nevertheless, the isolation of the viral causative agent remains a gold standard and a basic model for the study of genetic and antigenic changes in the virus population, as well as a means of detection of new respiratory viruses [12].

The mRT-PCR scores over the mono-plex rRT-PCR in a limited resource setting. The overall cost reduction may justify the routine use of these and rapid molecular diagnostic assays when compared with rRT-PCR. Such assays can actually be performed at least threefold lesser costs without compromising on quality. Importantly, the real time PCR machines are very expensive when compared to a conventional PCR.

Acknowledgments The authors are thankful to Ms. B Padmavathi Amma, Senior Technical Officer and her team, Advanced Clinical and Research Laboratory (ACRL), Government Medical College, Thiruvananthapuram, Kerala, India, for facilitating samples for this study.

\section{References}

1. Arnott A, Vong S, Sek M, Naughtin M, Beauté J, Rith S, Guillard B, Deubel V, Buchy P. Genetic variability of human metapneumovirus amongst an all ages population in Cambodia between 2007 and 2009. Infect Genet Evol. 2013;15:43-52.

2. Auburn H, Zuckerman M, Broughton S, Greenough A, Smith M. Detection of nine respiratory RNA viruses using three multiplex RT PCR assays incorporating a novel RNA internal control transcript. J Virol Methods. 2011;176:9-13.

3. Bellau-Pujol S, Vabret A, Legrand L, Dina J, Gouarin S, Petitjean-Lecherbonnier J, Pozzetto B, Ginevra C, Freymuth F. Development of three multiplex RT PCR assays for the detection of 12 respiratory RNA viruses. J Virol Methods. 2005;12:53-63.

4. Boivin G, Abed Y, Pelletier G, Ruel L, Moisan D, Cote S. Virological features and clinical manifestations associated with human metapneumovirus: a new paramyxovirus responsible for acute respiratory-tract infections in all age groups. J Infect Dis. 2002;186:1330-4.

5. Choudhary ML, Anand SP, Tikhe SA, Walimbe AM, Potdar VA, Chadha. Comparison of the conventional multiplex RT-PCR, real time RT-PCR and Luminex xTAG((R)) RVP fast assay for the detection of respiratory viruses. J Med Virol. 2016;188:51-7.

6. Ebihara T, Endo R, Kikuta H, Ishiguro N, Ishiko H, Hara M. Human metapneumovirus infection in Japanese children. J Clin Microbiol. 2004;42:126-32.

7. Fox JD. Nucleic acid amplification tests for detection of respiratory viruses. J Clin Virol. 2007;40:S15-23.

8. Freymuth F, Vabret A, Gouarin S, Petitjean J, Charbonneau P, Lehoux P. Epidemiology and diagnosis of respiratory syncitial virus in adults. Rev Mal Respir. 2004;21:35-42.

9. Ishioka T, Kimura H, Kita H, Obuchi M, Hoshino H, Noda M. Effects of respiratory syncytial virus infection and major basic protein derived from eosinophils in pulmonary alveolar epithelial cells (A549). Cell Biol Int. 2011;35:467-74.

10. Jain B, Singh AK, Dangi T, Agarwal A, Verma AK, Dwivedi M. High prevalence of human metapneumovirus subtype B in cases presenting as severe acute respiratory illness: an experience at tertiary care hospital. Clin Respir J. 2014;8:225-33.

11. Jokela P, Piiparinen H, Luiro K, Lappalainen M. Detection of human metapneumovirus and respiratory syncytial virus by duplex real-time RT-PCR assay in comparison with direct fluorescent assay. Clin Microbiol Infect. 2010;16:1568-73.

12. Jokela P, Piiparinen H, Mannonen L, Auvinen E, Lappalainen M. Performance of the luminex xTAG respiratory viral panel fast in a clinical laboratory setting. J Virol Methods. 2012;182:82-6.

13. Kim SR, Ki CS, Lee NY. Rapid detection and identification of 12 respiratory viruses using a dual priming oligonucleotide systembased multiplex PCR assay. J Virol Methods. 2009;156:111-6.

14. King AMQ, Lefkowitz E, Adams MJ, Carstens EB. Virus taxonomy classification and nomenclature of viruses ninth report of the international committee on taxonomy of viruses. New York: Elsevier Inc.; 2012.

15. Liolios L, Jenney A, Spelman D, Kotsimbos T, Catton M, Wesselingh S. Comparison of a multiplex reverse transcriptionPCR-enzyme hybridization assay with conventional viral culture and immunofluorescence techniques for the detection of seven viral respiratory pathogens. J Clin Microbiol. 2001;39:2779-83.

16. Litwin CM, Bosley JG. Seasonality and prevalence of respiratory pathogens detected by multiplex PCR at a tertiary care medical center. Arch Virol. 2014;159:65-72.

17. Mahony J, Chong S, Merante F, Yaghoubian S, Sinha T, Lisle C, Janeczko R. Development of a respiratory virus panel test for detection of twenty human respiratory viruses by use of multiplex PCR and a fluid microbead-based assay. J Clin Microbiol. 2007;45:2965-70.

18. Nair H, Nokes DJ, Gessner BD, Dherani M, Madhi SA, Madhi RJ. Global burden of acute lower respiratory infections due to respiratory syncytial virus in young children: a systematic review and meta-analysis. Lancet. 2010;375:1545-55.

19. Nunes IF, Moura FE. Isolation of respiratory syncytial virus from nasopharyngeal aspirates stored at $20{ }^{\circ} \mathrm{C}$ from one to fifteen months after collection. Mem Inst Oswaldo Cruz. 2006;101:451-3.

20. Tamura K, Stecher G, Peterson D, Filipski A, Kumar S. MEGA6: molecular evolutionary genetics analysis version 6.0. Mol Biol Evol. 2013;30:2725-9.

21. Vabret A, Sapin G, Lezin B, Mosnier A, Cohen J, Burnouf L, Petitjean J, Gouarin S, Campet M, Freymuth F. Comparison of three non-nested RT PCR for the detection of influenza-A viruses. J Clin Virol. 2000;17:167-75.

22. Van den Hoogen BG, de Jong JC, Groen J, Kuiken T, de Groot R, Fouchier RA, Osterhaus AD. A newly discovered human pneumovirus isolated from young children with respiratory tract disease. Nat Med. 2001;7:719-24.

23. van den Hoogen BG, van Doornum GJ, Fockens JC, Cornelissen JJ, Beyer WE, de Groot R, Osterhaus AD, Fouchier RA. Prevalence and clinical symptoms of human metapneumovirus infection in hospitalized patients. J Infect Dis. 2003;188:1571-7.

24. van Woensel JB, Bos AP, Lutter R, Rossen JW, Schuurman R. Absence of human metapneumovirus co-infection in cases of severe respiratory syncytial virus infection. Pediatr Pulmonol. 2006;41:872-4.

25. Wang W, Ren P, Sheng J, Mardy S, Yan H, Zhang J, Hou L, Vabret A, Buchy P, Freymuth F, Deubel V. Simultaneous detection of respiratory viruses in children with acute respiratory 
infection using two different multiplex reverse transcription-PCR assays. J Virol Methods. 2009;162:40-5.

26. Williams BG, Gouws E, Boschi-Pinto C, Bryce J, Dye C. Estimates of world-wide distribution of child deaths from acute respiratory infections. Lancet Infect Dis. 2002;2:25-32.
27. Williams JV, Martino R, Rabella N, Otegui M, Parody R, Heck JM. A prospective study comparing human metapneumovirus with other respiratory viruses in adults with hematologic malignancies and respiratory tract infections. $J$ Infect Dis. 2005;192:1061-5. 